\title{
Modeling the dynamics of a real biological system
}

\author{
I.O. Lixandru-Petre ${ }^{1 *}$ \\ ${ }^{1 *}$ Department of Automatic Control and Systems Engineering, University POLITEHNICA of Bucharest, ROMANIA \\ "Corresponding Author: E-mail: irinaoana.petre@gmail.com, Tel +40766596159 \\ ORCID iD: http:/orcid.org/0000-0003-4329-8911
}

\begin{abstract}
Infectious cells are and will continue to be a global problem, regarding the alteration of the health of individuals, the motivation for choosing this topic being the major implication the infectious cells has within the public health system and the way it acts, managing to successfully overcome barriers which the body raises to maintain cellular stability. The article brings new contributions related to the topic of dynamic modeling the immunity system, by determining a mathematical model of the interaction between the cells of the immune system and the cells of the infectious agent. Different types of behaviors are presented taking into account the entry of viral particles into the target cells, behaviors of the model without delay time, followed by time predictions for the delay time model. Finally, we compared the models and concluded that the delay time model is much more realistic, through the oscillations and fluctuations in the three components in the primary stages of infection.
\end{abstract}

Keywords: infectious, model, dynamic modeling, simulation.

DOI: http://dx.doi.org/10.4314/ijest.v13i2.5

Cite this article as:

Lixandru-Petre I.O. 2021. Modeling the dynamics of a real biological system. International Journal of Engineering, Science and Technology, Vol. 13, No. 2, pp. 42-49. doi: $10.4314 /$ ijest.v13i2.5

Received: May 16, 2021; Accepted: May 28, 2021; Final acceptance in revised form: June 1, 2021

\section{Introduction}

Cellular biological systems are characterized by continuous exchanges of signals with the environment, this feature being essential for the existence and proper functioning of organisms. Alterations in the cellular system can have serious consequences on cellular stability and can lead to the formation of viruses (Alberts et al., 2002). This paper addresses the problem of infectious cells formation, in terms of a virus that manipulates the apoptotic pathway to ensure the perpetuation of its own survival, inhibiting the apoptosis of infected cells, allowing viral replication, activating only that of host cell defense mechanisms. For example, by destroying or damaging cells in the immune system, the human immunodeficiency virus (HIV) progresses, destroying the body's ability to fight infections. The HIV virus differs from others in that it does not have the ability to reproduce independently, needing a host to support reproduction. It inserts into the host's DNA - copies of their DNA, so that when the cell is stimulated to reproduce, it also reproduces copies of the virus, progressively compromising the host's autoimmune response, leading to loss of cellular function and host susceptibility to opportunistic infections (Alberts et al., 2002).

HIV belongs to a class of viruses called retroviruses, which represent viruses that contain RNA as genetic material. After infection of the cell, HIV uses an enzyme called reverse transcriptase to convert RNA into DNA, continuing its replication using cell reproduction. HIV or immunodeficiency virus attacks the immune system by destroying T-cells (CD4 +), a type of white blood cell that is vital in destroying and overcoming infections (Alberts et al, 2002). The destruction of these cells leaves people 
infected with HIV vulnerable to infections, diseases and other complications. The HIV virus does not have the ability to reproduce independently, so it needs a host to support reproduction. HIV infects white blood cells with the RNA virus which, once inside the cell, leads to the formation of a copy of its genome, which is inserted into the genome of the host cell, which can cause AIDS syndrome.

In short, the interaction between the virus and white cells (CD4 immune system cells) takes place with the help of the envelope glycoprotein of the virus-gp120 (Alberts et al., 2002). CD4 + T cells represent white blood cells, with a key role in maintaining the integrity of the function of the human immune system, through the immune system understanding all the mechanisms of the human body to fight infections and to eliminate them and other dysfunctional cells. They organize the immune system's response to certain microorganisms, including bacteria, fungal infections, and viruses, being vital in ensuring the proper functioning of the body Alberts et al., 2002). Gp 120 is an envelope protein responsible for virus entry into the host cell, so the glycoprotein on the HIV envelope binds to CD4 receptors and allows viral cells to enter the immune system cells, leading to virion proliferation and cell infection (Alberts et al., 2002).

\section{Approach}

Models are more or less detailed representations of ideas, phenomena, processes, which take various forms, such as sketches, graphs, or differential equations. They are more or less successful approximations of the real world, with a certain utility or purpose; most of the time, these being descriptions of the functionalities of certain real processes (Buiu et al., 2011). The mathematical model is a system of mathematical relations, which describes the essential properties of the original. Solving a real problem can be reduced to solving a mathematical problem, mathematical symbolism being one of the most commonly used means for describing the properties of the surrounding world, primarily quantitative ones (Hinchliffe, 2008). In mathematical models, a particular type of model is represented by simulation models, which simulate the phenomena that characterize the real system, studying the behavior of the system, as it evolves over time, in the form of a set of hypotheses expressed by relationships mathematical, logical and symbolic (Holtje et al., 2008).

Dynamic models are those that take into account changes in the system over time (Ramachandran et al., 2008). A continuous system is one in which the state variables change continuously over time, and a continuous model will be the one that approximates the real system, with a certain purpose; most of the time, this is to describe the functionality of the real process. Exploiting molecular biology can be greatly facilitated by molecular-based modeling (Van Gunsteren et al., 2006). A robust review containing developments in HIV modeling is provided in Perelson and Ribeiro (2013). Balasubramaniam et al. (2012) provides useful information on the analysis of the stability and Hopf bifurcation of a mathematical model of HIV infection of Tcells, while Banks et al. (2008) provides a system of ordinary differential equations to describe the pathogenesis of HIV infection. Kwon et al. (2012) proposes an age-structured model allowing for variations in the virion production rate and the death rate of infected $\mathrm{T}$ cells towards (Yuzbasi and Ismailov, 2017) which considers a different model for the human immunodeficiency virus (HIV) infection - CD4+T-cells. Mircea et al. (2006) provides a mathematical model with delay kernel for the role of the immune response time delay in periodic therapy of the tumors, while Pawelek et al. (2012) introduces a model with two delays, fitting it to viral load datafrom 10 patients during primary HIV-1 infection.

Next, we will mathematically model the dynamics of the HIV virus, a model that describes the dynamics of the interaction between pathogens and the immune system. The purpose of the model is to study the properties and transformations of a real biological process, subjecting the model to diverse influences. Initially, the model will be one without periods of time to express the duration between the moment the virions enter the cell and the actual replication in the host cell, and later to incorporate into the model periods of delay, to observe the behaviors that this virus has on cells. during the latency period (such as a decrease in the number of CD4 cells), the new model leading to a better understanding of HIV behavior.

A schematic representation of the real biological system is presented in Figure 1:

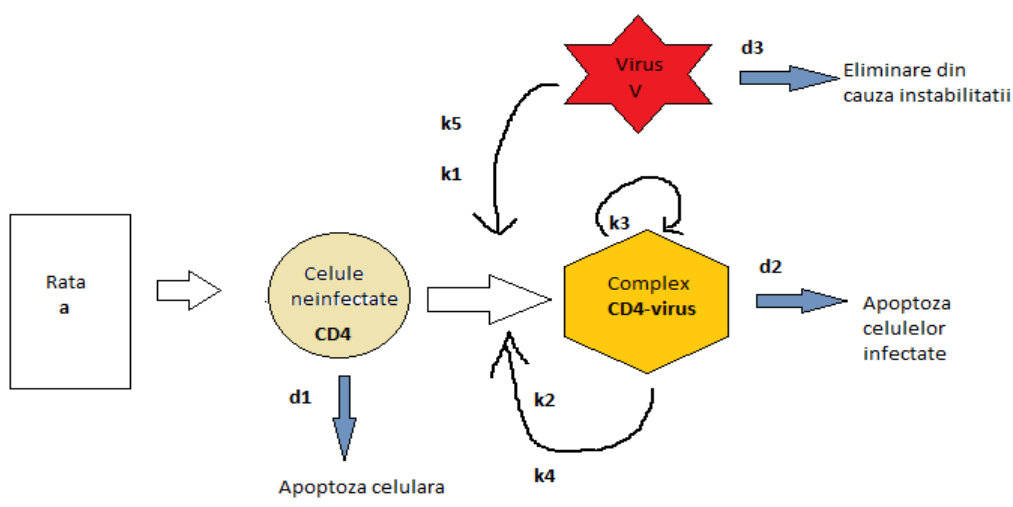

Figure 1. Graphic model suggestive of the interaction between immune system cells and virus 
Apart from the model proposed by the schematic representation, the process can also be described by means of the mathematical relations of the reactions that take place within this system (Ramachandran et al., 2008). To begin with, we made objective assumptions about the process, choosing for mathematical representation only three equations specific to the three vital types of behavior in describing biological events (ignoring other classes of cells that did not provide information about HIV dynamics). Delay was defined taking into account the behaviors of uninfected cells, infected cells, and free virions. These behaviors describe population dynamics, forming a system of 3 differential ordinary equations presented below.

For simplicity of writing, notations will be used for certain terms, namely: CD4 the white blood or target cells notated with T, viral particles with $\mathrm{V}$ and the CD4-virus complex with I from infected cells.

The model without delay times is a continuous model expressed by a system of differential equations, of the form:

$$
\left\{\begin{array}{c}
\frac{d T}{d t}=a-d_{1} * T-k_{1} * V * T-k_{2} * I * T \\
\frac{d I}{d t}=k_{1} * V * T+k_{2} * I * T-d_{2} * I \\
\frac{d V}{d t}=k_{2} * I+k_{4} * I * T-d_{3} * V-k_{5} * V * T
\end{array}\right.
$$

where $k_{1} \div k_{5}$ represents the kinetic coefficients of the system; the rate constants that describe the rates of formation of the various components or resulting compounds, $d_{1} \div d_{3}$ represent the rates of exit from the system of the three types of behaviors, and the constant $\alpha$ represents the rate of reproduction outside the system of immune system cells, i.e. the number of cells CD4 that already exists in the body before the virus enters.

Assumptions made:

- target cells are produced with a constant rate of $a$, die at the rate of $d_{1}$, are infected by free virions with the rate of $k_{1}$ and by infected cells (if they already exist in the body) with the rate of $k_{2}$;

○ the interaction of immune system cells and viral cells lead to the birth of infected cells, which die at the rate of $d_{2}>d_{1}$, reflecting the shortening of the life of infected cells and are produced by the interaction between target cells and previous infected cells or virions at rates $k_{2}$, respectively $k_{1}$;

- Free virions are produced by infected cells at the rate of $k_{3}$, by the complex of CD4-gp120 cells at the rate of $k_{4}$ and leave the system at the rate of $d_{3}$, and $k_{5}$ being eliminated by the interaction between CD4 cells and existing virions;

The delayed model has different dynamics than the first because it can lead to population fluctuations or system instability. Scientists believe that there are 2 types of delay periods: the first that occurs between contact with the virus and its appearance in cells and the second-delay between the initial HIV infection and the creation of new virions.

In this paper, we incorporated an intracellular delay into the model to describe the time period between the time of the initial HIV infection of the host cell and the release of new virions into the body, resulting in a system of delayed differential equations. Continuous model expressed by the same system of differential equations, but with delay times:

$$
\left\{\begin{array}{c}
\frac{d T}{d t}=a-d_{1} * T-k_{1} * V(t-\tau) * T(t-\tau)-k_{2} * I * T \\
\frac{d I}{d t}=k_{1} * V(t-\tau) * T(t-\tau)+k_{2} * I * T-d_{2} * I \\
\frac{d V}{d t}=k_{2} * I+k_{4} * I * T-d_{3} * V-k_{5} * V(t-\tau) * T(t-\tau)
\end{array}\right.
$$

The assumptions made are identical, $k_{1} \div k_{4}$ represent the same kinetic coefficients of the system, and $d_{1} \div d_{2}$ represent the same exit rates from the system of the three types of behaviors. The constant $\alpha$ represents the rate of reproduction outside the system of the cells of the immune system, before the penetration of the virus. The only change is the occurrence of the delay time $\tau$ in the interaction between target cells and virions. Discrete intracellular delay $(\tau)$ was introduced to describe the time required for infected cells to produce virions after viral entry, in order to study the effects that the delay period has on system dynamics and stability. It was chosen equal to one day, the studies concluding that the duration of viral production is $\leq 1.5$. 


\section{Discussion and results}

For the graphical representation of the continuous model without delay time and solving the system of nonlinear differential equations, expressed by (2), the Matlab ode45 function is used, a solver that calculates the values of the functions, obtained by integrating differential equations, in the points received as a parameter, using the numerical technique of order 4 and 5 of RungeKutta type. For the results obtained below, we used values of the kinetic constants, as well as of the production rate outside the system, set at choice, in order to demonstrate the principle of the system. Thus, for different values associated with the model parameters, we obtained various behaviors.

For example, in the graphs in Figure 2, it is detected an increase in the concentration of healthy cells until an equilibrium is reached, thus keeping the cells in control and normal proliferation, while the concentration of infected cells and of virions decreases due to the values of the increased concentration of healthy cells. In this case, the immune system is strong enough to fight and win the "battle" with the viral cells.
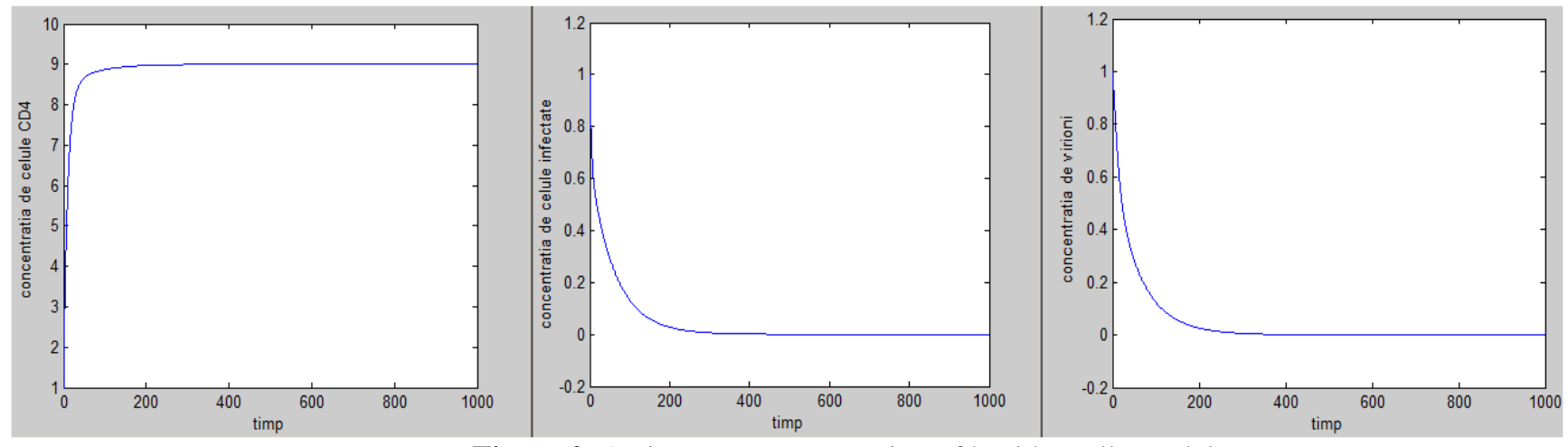

Figure 2. An increase concentration of healthy cells model

The graphs in Figure 3. show some changes compared to the first sets of graphs, such cases could be explained when the death rate of target cells is very low, the rate of infected cells is high due to virions that destroy the cell, and viral cells they have a higher rate than the target cells, but lower than the infected cells.
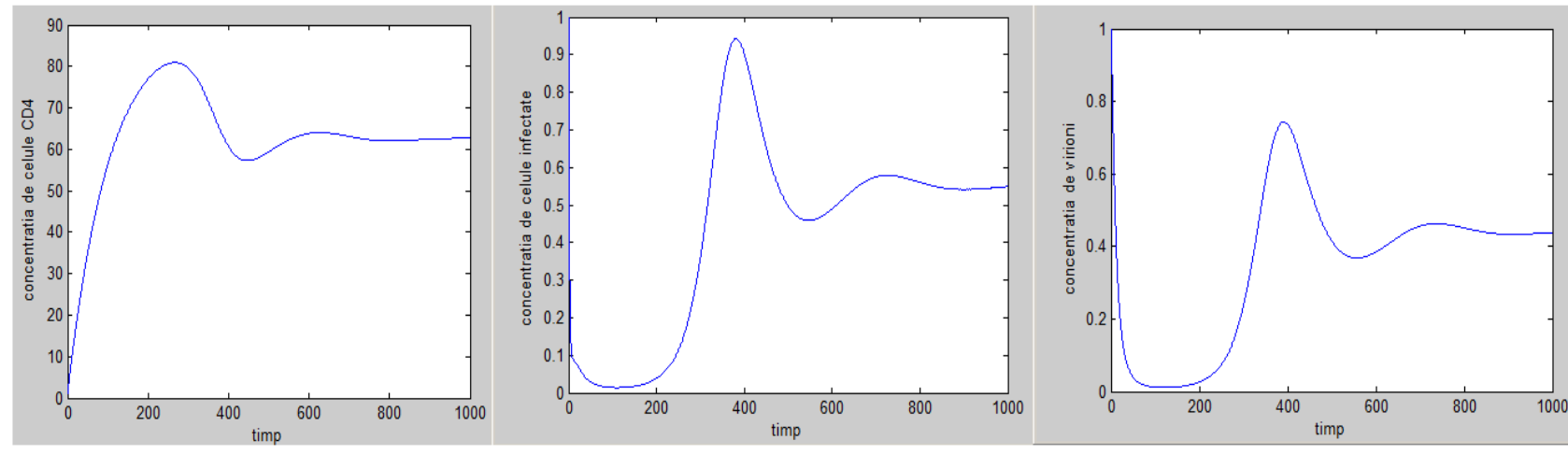

Figure 3. A desirable behavior in terms of death rates for HIV modelling

This kind of behavior would be desirable - in terms of death rates. Once the disease is known, although viral entry has taken place, immediate treatment could stop the spread of virions and lead to a recovery of the immune system. On the other hand, if the body is weakened, the individual is susceptible to several diseases, and the body will respond much more slowly to treatment, so there is a possibility that the immune system will not recover. For the graphical representation of the continuous model with delay times, expressed by (3), the Matlab dde23 function will be used. Equations can no longer be solved with ode 45, because the value of the solution at time $t$ depends on the value of the solution at time $\tau$ last, the function dde 23 being the one that solves these types of differential equations with constant delay times.

For the results obtained below, we also used values of the parameters set at choice, in order to demonstrate the principle of the system, obtaining various behaviors. In Figure 4, we can see an initial increase in the concentration of healthy cells, followed by a decrease in their stabilization, while the concentration of infected cells as well as that of virions increases due to the values of low cell concentration. In this case, the immune system is weakened and any additional infection caused by the disease can be fatal. 

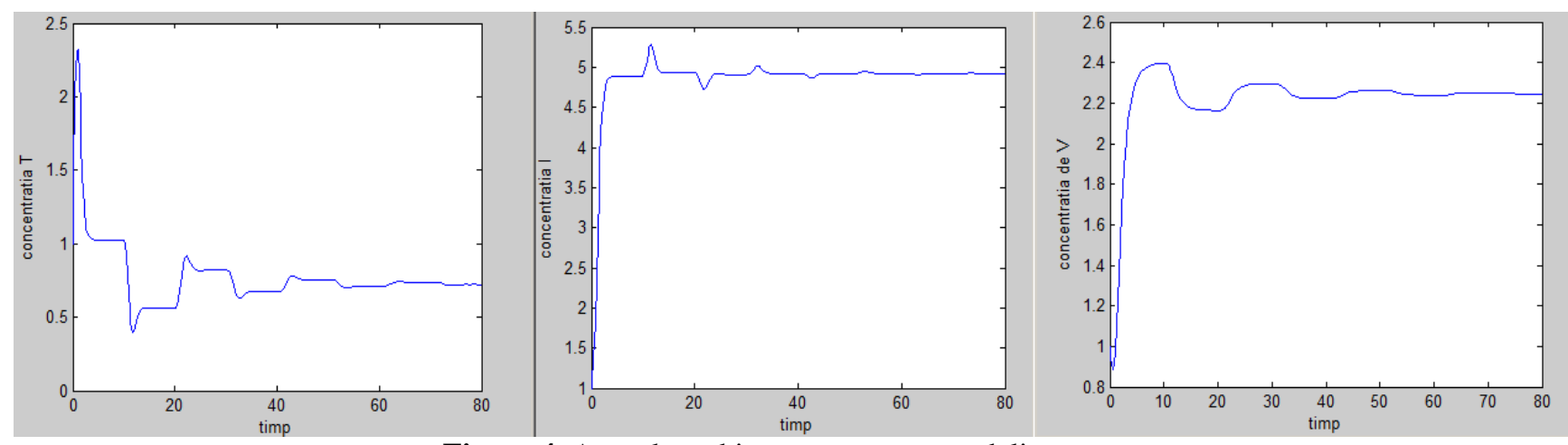

Figure 4. A weakened immune system modeling case

The graphs in Figure 5. show some changes from the sets of graphs above, due to the oscillating responses of the behaviors.
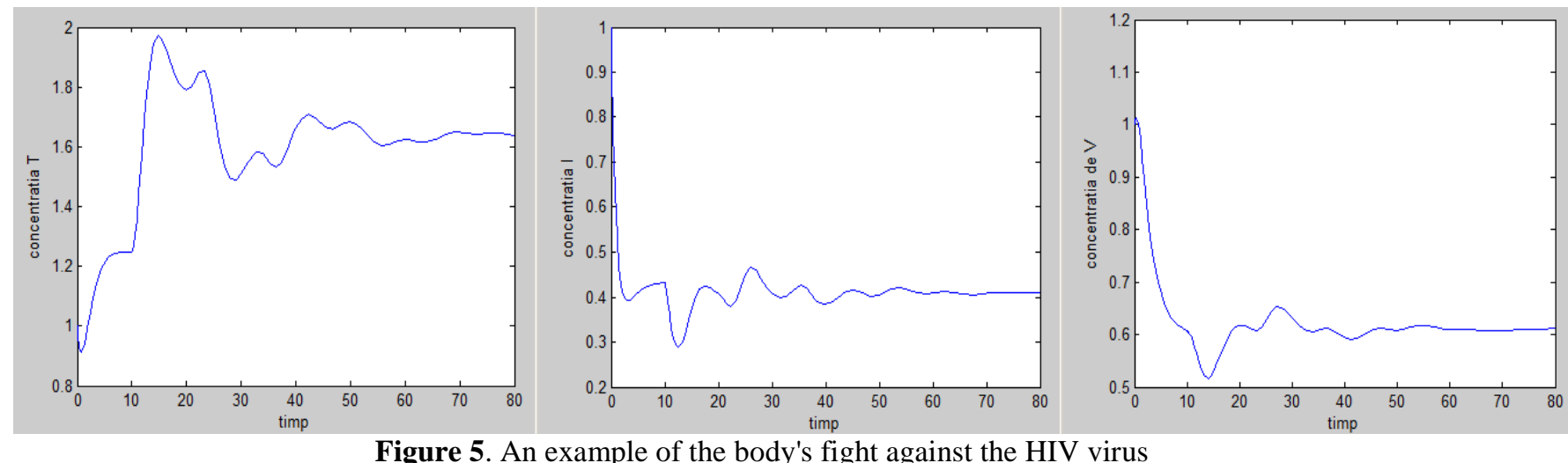

Figure 5. An example of the body's fight against the HIV virus

And in reality, these behaviors occur in HIV-positive patients and signify the body's fight against the virus to defend the immune system. This is a happy case in which CD4 cells manage to grow to the detriment of infected ones and released virions. Treatment is the one that decreases the number of infected cells and increases the number of CD4 cells, stopping the progression of the infection and thus reducing the death rates. For the simulation part, several categories of disease behaviors were determined and displayed graphically for both models without and with delay.

Thus, for a small number of healthy cells existing in the system and for $k_{2}>k_{1}$ (i.e. for a larger number of target cells interacting with infected cells against viruses) the behavior of Figure 6. is obtained, where we can conclude that for the patient, once the virus enters the body, its cell proliferation begins by increasing the number of infected cells and thus the number of virions. Detected, the virus will be able to be treated with drugs that reduce the number of infected cells and virions, keeping concentrations constant, thus "helping" CD4 cells to grow in number.
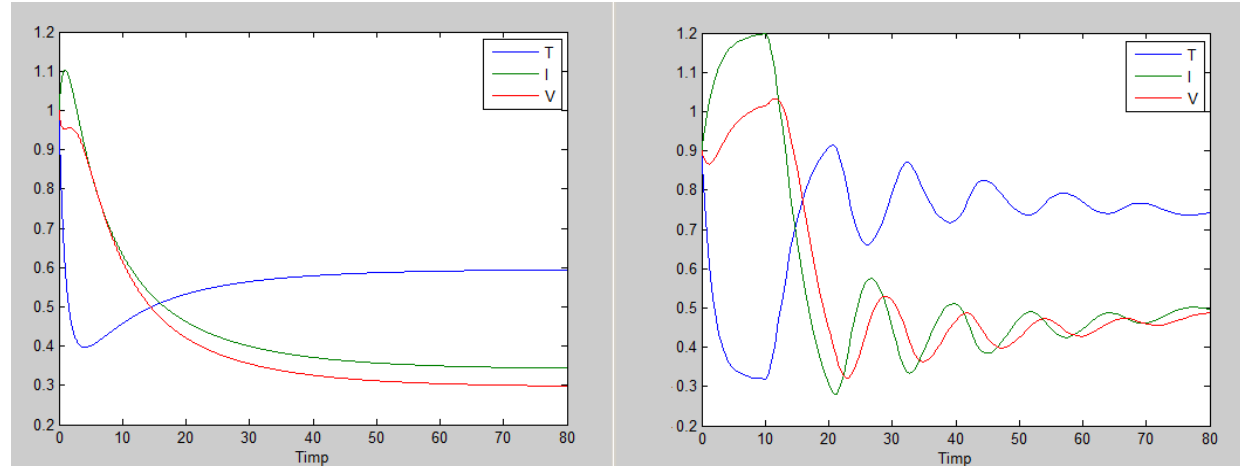

Figure 6. Case modeling without and with delay for $k_{2}>k_{1}$

Different from the previous behavior by a larger number of CD4 cells that were already in the body at the time of infection, Figure 7 illustrates a case of a patient infected with HIV who begins treatment, succeeding in a similar way, to stop cell proliferation of virions, maintaining a constant number of viral particles, having control over the disease. 


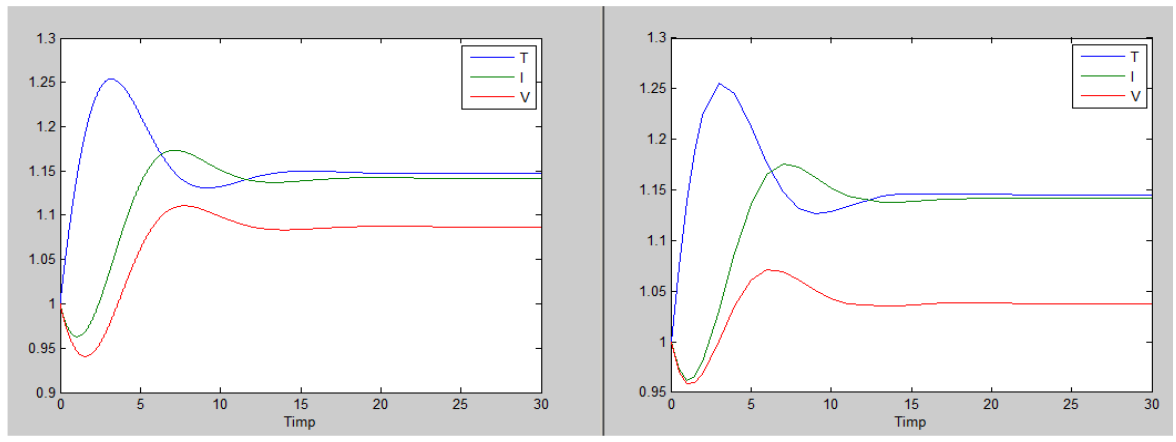

Figure 7. Case modeling of control over the disease (without and with delay model)

The graphs in Figure 8 shows the case in which the number of virions exceeds the number of CD4 cells, the patient is in a more advanced stage of the disease, fluctuations of all three types of behavior occur, but eventually (after about 150 days) the system will stabilize and the disease will be kept under control.
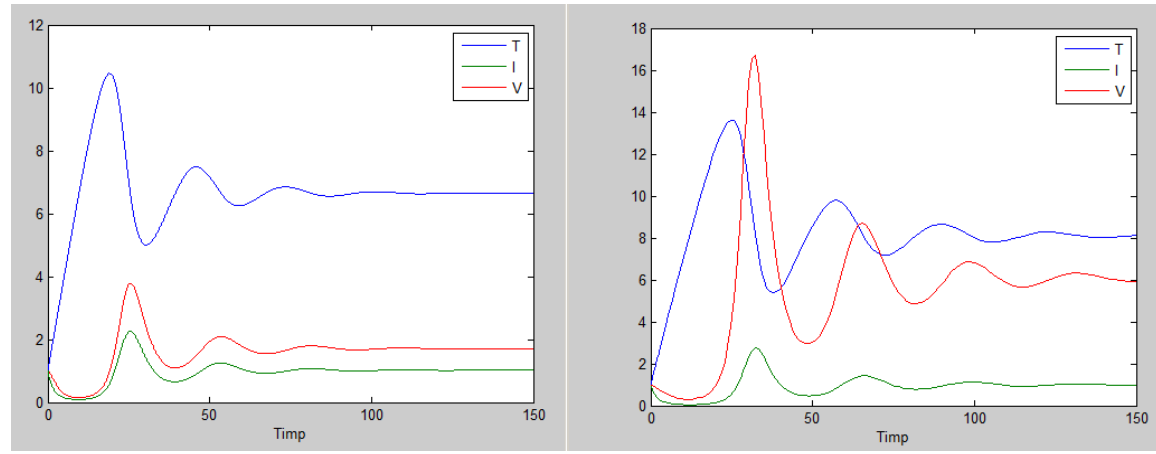

Figure 8. A case with number of virions >> the number of CD4 cells

There are also cases, such as Figure 9, in which $k_{3}>a$, where the system response to the two types of models is almost identical. In this example, the patient begins treatment, which stops the proliferation of infected cells, but existing virions and those that interact with host cells continue to reproduce, reaching a very large number, the disease can no longer be controlled, any other new infection can be fatal.

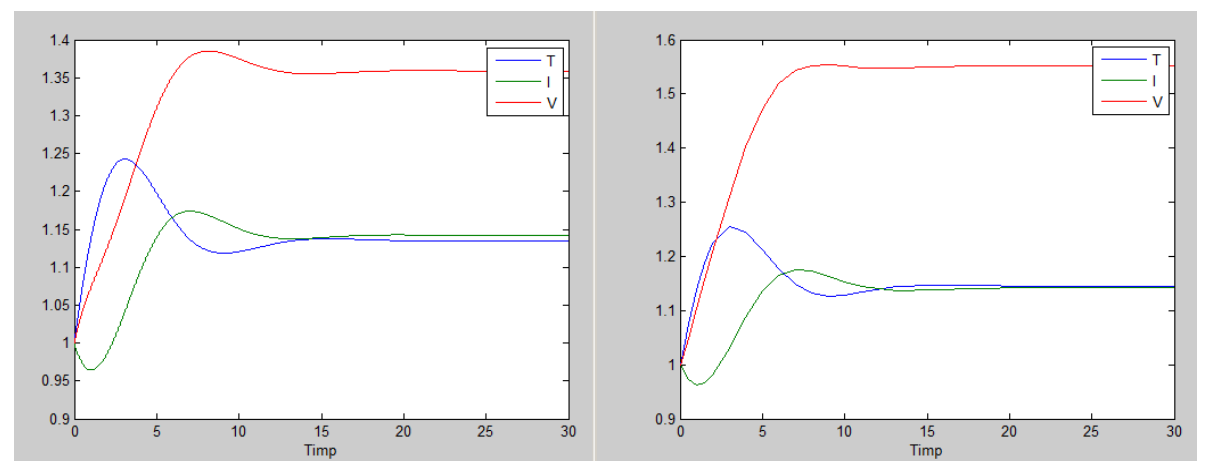

Figure 9. Case modeling without and with delay for $k_{2}>$ a

\section{Conclusions}

There are currently over 30 million people living with HIV worldwide. Every 6 seconds a new person becomes infected, and it is estimated that by the end of 2021 there will be more than 50 million HIV-infected people worldwide. HIV or immunodeficiency virus is the one that attacks the immune system by destroying T cells (CD4 +), a type of white blood cell that is vital in destroying and overcoming infections. The destruction of these cells leaves people infected with HIV vulnerable to infections, diseases, and other complications. It should be noted that the human immunodeficiency virus (HIV) is transmitted, while AIDS is not transmitted. AIDS is a set of signs and symptoms, a final stage of infection. The $120 \mathrm{gp}$ protein on the HIV envelope binds to CD4 
receptors, and after the intracellular delay associated with the production of viral protein capsids, infected cells release hundreds of virions that affect $\mathrm{T}$ cells. Based on this biological information, we studied the dynamics of the virus through a model with and without delays, concluding that the system is asymptotically stable for both types of models, the latter being much more realistic, through oscillations and fluctuations in the three components in the primary stages of infection.

The aim of this paper was to dynamically model the interaction between immune system cells and HIV cells, in order to obtain information on the processes and overall behavior of the system, as they evolve overtime, the prediction over time being in my opinion, the simplest way for an infected patient to understand the stage of his disease. The mathematical equations allowed the voluntary modification of the different values of the parameters apart from those already obtained from clinical analyzes and thus, the submission of the model to various influences.

The originality of this work consists precisely in modeling the biological process that involves the interaction between virions and human cells and the schematic reproduction of the real system in the form of a similar system. The process of viral entry into the body was simulated, based on graphs obtaining predictions over time on the system. The biological process described and modeled plays a very important role in the best possible understanding of how viral cells interact with white ones, the concentrations of each cell type in the body having a vital role in keeping the disease under control or not.

Although much remains unknown about this problem (high mutation rate, rapid replication cycle, and long, individual-toindividual infection duration), new information on the dynamic replication of the immune virus is extracted from various mathematical models, helping researchers to develop new anti-infectious research projects, mathematical modeling contributing to a better understanding of the fundamentals of human-viral cell interaction, and may suggest new ways to monitor and improve current treatments, studies on the dynamics of the human autoimmune virus attracting more and more attention lately.

A potential direction of research related to the topic presented may be designing and implementing a diagnostic system in medicine based on agents, a system that, according to different key concepts (e.g. clinical data, hereditary data, age), is able to make a decision about a type of treatment after identifying a particular disease or stage of the disease.

\section{Acknowledgement}

I.-O. Lixandru-Petre would like to thanks C. Buiu and R. Mihu for all the support they give for all the manuscripts and ideas.

\section{References}

Alberts B., Johnson A., Lewis J., Raff M., Roberts K., and Walter P., 2002, Molecular Biology of the Cell, 4th edition, Garland Science, New York

Balasubramaniam P., Prakash M., Park J.H., 2012, Hopf bifurcation analysis of delayed model of thymic infection with HIV-1, Applied Mathematics and Computation, Vol. 218, No. 23, pp. 11505-11517. https://doi.org/10.1016/j.amc.2012.05.038

Banks H.T., Davidian M., Hu S., Kepler G.M., Rosenberg E.S., 2008, Modeling HIV immune response and validation with clinical data, Journal of Biological Dynamics, Vol. 2, No. 4, pp. 357-385. doi: 10.1080/17513750701813184

Buiu C., Pavel A., Vasile C., 2011, Biomatematică şi Bioinformatică: Concepte şi Aplicaţii, Editura Universitară, Bucuresti, Romania.

Hinchliffe A., 2008, Molecular Modelling for Beginners, Wiley-Interscience, New York.

Holtje H.D., Sippl W., Rognan D., Folkers G., 2008, Molecular Modeling: Basic Principles and Applications, $3^{\text {rd }}$ Edition, Pharmaceutical \& Medicinal Chemistry, Wiley-Interscience, New York.

Hünenberger P.H., Kastenholz M.A., Oostenbrink C., Schenk M., Trzesniak D., van der Vegt N.F.A., Yu H.B. 2006. Biomolecular modeling: Goals, problems, perspectives. Journal of Computational Chemistry, Vol. 45, pp. 4064-4092. doi: 10.1002/anie.200502655.

Kwon H., Lee J., Yang S.D. 2012, Optimal control of an age-structured model of HIV infection, Applied Mathematics and Computation, Vol. 219, No. 5, pp. 2766-2779. https://doi.org/10.1016/j.amc.2012.09.003

Mircea G., Neamţu M., Horhat R.F., Opris D. 2006. A mathematical approach with delay kernel for the role of the immune response time delay in periodic therapy of the tumors. Conference Francophone sur la Modelisation Mathematique en Biologie et en Medecine, Craiova, Romania,12-14 July, pp. 1-22.

Pawelek K.A., Liu S., Pahlevani F., Rong L. 2012, A model of HIV-1 infection with two time delays: Mathematical analysis and comparison with patient data, Mathematical Biosciences, Vol. 235, No. 1, pp. 98-109. https://doi.org/10.1016/j.mbs.2011.11.002

Perelson A.S., Ribeiro R.M. 2013, Modeling the within-host dynamics of HIV infection. BMC Biology, Vol. 11, Article 96. https://doi.org/10.1186/1741-7007-11-96

Ramachandran K.I., Gopakumar D., Namboori K., 2008, Computational Chemistry and Molecular Modeling: Principles and Applications, Theoretical and Computational Chemistry, Springer Nature, Switzerland.

van Gunsteren W.F., Bakowies D., Baron R., Chandrasekhar I., Christen M., Daura X., Gee P., Geerke D.P., Glättli A.,

Yüzbaş1 Ş., Ismailov N. 2017. A numerical method for the solution of the HIV infection model of CD4 T cells.

International Journal of Biomathematics, Vol. 10, No. 7, https://doi.org/10.1142/S179352451750098X. 


\section{Biographical notes}

I.-O. Lixandru-Petre is Ph.D. student at Politehnica University of Bucharest, Romania, Dept. of Automatic Control and Systems Engineering. She received a master's degree in "Intelligent driving systems" also at Politehnica University of Bucharest and from 2014 when she starts her Ph.D., being interested in decision support systems and gene expression analysis. From 2012 until 2020, she was assistant for Diagnostic and Decision techniques laboratory, working with techniques and methods like Naive Bayes, Decision Tree or Bayesian networks. 